Phys. Rev. Lett. (June 3, 1996) in press.

\title{
Isospin dependence of collective flow in heavy-ion collisions at intermediate energies
}

\author{
Bao-An Li ${ }^{a}$, Zhongzhou Ren ${ }^{b, c}$, C.M. Ko ${ }^{a}$ and Sherry J. Yennello ${ }^{d}$ \\ a Cyclotron Institute and Department of Physics \\ Texas A\&MM University, College Station, TX 77843, USA \\ b Ganil, BP5027, F14021 Caen Cedex, France \\ c Department of Physics, Nanjing University, Nanjing 210008, P.R. China \\ d Cyclotron Institute and Department of Chemistry \\ Texas A\&MM University, College Station, TX 77843, USA
}

\begin{abstract}
Within the framework of an isospin-dependent Boltzmann-Uehling-Uhlenbeck (BUU) model using initial proton and neutron densities calculated from the nonlinear relativistic mean-field (RMF) theory, we compare the strength of transverse collective flow in reactions ${ }^{48} \mathrm{Ca}+{ }^{58} \mathrm{Fe}$ and ${ }^{48} \mathrm{Cr}+{ }^{58} \mathrm{Ni}$, which have the same mass number but different neutron/proton ratios. The neutron-rich system $\left({ }^{48} \mathrm{Ca}+{ }^{58} \mathrm{Fe}\right)$ is found to show significantly stronger negative deflection and consequently has a higher balance energy, especially in peripheral collisions.
\end{abstract}


Nuclear collective flow in heavy-ion collisions at intermediate energies has been a subject of intensive theoretical and experimental studies during the last decade, for a general introduction and overview see [1]. The study of the dependence of collective flow on entrance channel parameters, such as, the beam energy, mass number and impact parameter, have revealed much interesting physics about the properties and origin of collective flow. In particular, by studying the beam energy dependence it has been found that the transverse collective flow changes from negative to positive at an energy $E_{b a l}$ (defined as the balance energy) due to the competition between the attractive nuclear mean field at low densities and the repulsive nucleon-nucleon collisions [2 10]. The balance energy was found to depend sensitively on the mass number, impact parameter and properties of the colliding nuclei, such as the thickness of their surfaces [11]. Furthermore, detailed theoretical studies mainly using transport models (for a review see e.g. [12 14]) have shown that both the strength of transverse flow and the balance energy can be used to extract information about the nuclear equation of state and in-medium nucleon-nucleon cross sections (e.g. [15 26]).

With high intensity neutron-rich or radioactive beams newly available at many facilities, effects of the isospin degree of freedom in nuclear reactions can now be studied in more detail for a broad range of beam energies and projectile-target combinations (e.g. [27,28]). These studies will put stringent constraints on the isospin-dependent part of nuclear equation of state. The latter is vital for determining, for example, the maximum mass, moment of inertia and chemical composition of neutron stars [29], where in the crust neutron-rich nuclei coexist with a gas of free neutrons and in the core the isospin dependence of the nucleon-nucleon interaction determines the stiffness of the equation of state [30,31]. In this Letter we report results of the first theoretical study on the isospin dependence of transverse flow in heavy-ion collisions at intermediate energies. A strong isospin dependence of the transverse flow was found at energies around and below the balance energy, especially in peripheral collisions. An experimental study of the isospin dependence of transverse collective flow will soon be carried out at NSCL/MSU [28]. Detailed comparisons between experimental data and model predictions in the future will shed light on the form and strength of the isospin-dependent 
part of nuclear equation of state, the isospin-dependent in-medium nucleon-nucleon cross sections, and the properties of neutron-rich nuclei.

In this study we use a Boltzmann-Uehling-Uhlenbeck (BUU) transport model which includes explicitly isospin degrees of freedom. The model has been used recently to explain successfully several phenomena in heavy-ion collisions at intermediate energies which depend on the isospin of the reaction system [32,33]. The isospin dependence comes into the model through both the elementary nucleon-nucleon cross sections $\sigma_{12}$ and the nuclear mean field $U$. Here we use the experimental nucleon-nucleon cross sections with explicit isospin dependence [34]. We keep in mind, however, that in-medium cross sections and their isospin dependence might be strongly density dependent [35,36]. The nuclear mean field $U$ including the Coulomb and isospin symmetry terms is parameterized as

$$
U\left(\rho, \tau_{z}\right)=a\left(\rho / \rho_{0}\right)+b\left(\rho / \rho_{0}\right)^{\sigma}+\left(1-\tau_{z}\right) V_{c}+C \frac{\rho_{n}-\rho_{p}}{\rho_{0}} \tau_{z}
$$

In the above, $\rho_{0}$ is the normal nuclear matter density; $\rho, \rho_{n}$ and $\rho_{p}$ are the nucleon, neutron and proton densities, respectively; $\tau_{z}$ equals 1 or -1 for neutrons or protons, respectively; and $V_{c}$ is the Coulomb potential. We use the so-called soft equation of state with a nuclear compressibility of $K=200 \mathrm{MeV}$ and a value of $32 \mathrm{MeV}$ for the strength $C$ of the symmetry potential.

For our first exploratory study on the isospin dependence of the collective flow we select two reaction systems, ${ }^{48} \mathrm{Cr}+{ }^{58} \mathrm{Ni}$ and ${ }^{48} \mathrm{Ca}+{ }^{58} \mathrm{Fe}$, which have the same mass number of $48+58$ but different neutron/proton ratios of 1.04 and 1.30, respectively. The neutron and proton distributions for these nuclei are determined from the well-known relativistic meanfield (RMF) theory (e.g. [37]). More specifically, we use a version of the theory with the NL$\mathrm{SH}$ force parameters [38]. The theory describes very well the ground state properties of nuclei near and far away from the stability line. In particular, it provides a very good description of the neutron-skin thickness of nuclei with large neutron excesses 38 40. The neutron, proton and matter densities calculated for the four nuclei studied here are shown in Fig. 1 and Fig. 2. It is seen that there are clear neutron skins for the two neutron-rich nuclei ${ }^{48} \mathrm{Ca}$ and 
${ }^{58} \mathrm{Fe}$. While the matter densities $(\mathrm{n}+\mathrm{p})$ in ${ }^{48} \mathrm{Ca}$ and ${ }^{48} \mathrm{Cr}$ are almost identical, the matter density in ${ }^{58} \mathrm{Fe}$ is more extended than in ${ }^{58} \mathrm{Ni}$. The calculated charge densities of these nuclei are actually very close to those measured from electron scattering experiments [41]. In the BUU model, we then initialize the spacial coordinates of neutrons and protons in the four nuclei according to the calculated densities. The momentum distributions of nucleons are generated using the local Thomas-Fermi approximation. It is worth mentioning that one can also initialize the neutron and proton distributions by running the Vlasov mode of the BUU model for each nucleus. Indeed, certain neutron skins can be produced for heavy nuclei by using a strong symmetry potentials within the Vlasov model [32,33,42,43]. Nevertheless, the approach used in the present study is much more reliable in terms of reproducing the ground state properties of neutron-rich nuclei.

The standard transverse momentum analysis [15] (see also [1]) was performed for the two reaction systems. Typical results for central collisions at an impact parameter of $2 \mathrm{fm}$ and beam energies of 50, 60 and $70 \mathrm{Mev} /$ nucleon are shown in Fig. 3. At a beam energy of $50 \mathrm{MeV} /$ nucleon, the transverse flow in the reaction of ${ }^{48} \mathrm{Ca}+{ }^{58} \mathrm{Fe}$ is still negative while that in the reaction of ${ }^{48} \mathrm{Cr}+{ }^{58} \mathrm{Ni}$ is already positive. The difference disappears at beam energies above $70 \mathrm{MeV} /$ nucleon. To be more quantitative we have extracted the flow parameter $F$ defined as the slope of the transverse momentum distribution at the center of mass rapidity $y_{\mathrm{cm}}$. The beam energy dependence of the flow parameter for the two reaction systems at impact parameters of $2 \mathrm{fm}$ and $5 \mathrm{fm}$ are shown in Fig. 4 . The lines are the least-square fits to the calculations using linear functions $F(C a+F e)=-32.2+0.55 E / A$ and $F(C r+N i)=-23.9+0.48 E / A$ at $\mathrm{b}=2 \mathrm{fm} ;$ and $F(C a+F e)=-35.9+0.22 E / A$ and $F(C r+N i)=-23.2+0.18 E / A$ at $\mathrm{b}=5 \mathrm{fm}$. It is seen that in both central and peripheral collisions the neutron-rich system ${ }^{48} \mathrm{Ca}+{ }^{58} \mathrm{Fe}$ shows systematically smaller flow parameters indicating a stronger attractive interaction during the reaction. The effect is more appreciable in peripheral collisions as one expects. Consequently, the balance energy in ${ }^{48} \mathrm{Ca}+{ }^{58} \mathrm{Fe}$ reaction is higher than that in the reaction of ${ }^{48} \mathrm{Cr}+{ }^{58} \mathrm{Ni}$ by about 10 to $30 \mathrm{MeV} /$ nucleon. The difference between flow parameters in the two systems decreases as 
the beam energy increases and finally disappears as the beam energy becomes far above the balance energy.

The observed isospin dependence of the collective flow is a result of the competition among several mechanisms in the reaction dynamics. First, it is well known that nucleonnucleon collisions cause repulsive collective flow, and this effect is proportional to the number of collisions in the overlapping volume. While the number of particles in this volume in the two reaction systems is roughly the same, the number of collisions in the reaction of two neutron-rich nuclei is smaller since the neutron-neutron cross section is about a factor of three smaller than the neutron-proton cross section in the energy region studied here. This effect is stronger in peripheral collisions where two thick neutron skins are overlapping during the reaction of two neutron-rich nuclei. Second, the Coulomb potential also causes repulsive scatterings. This effect is obviously weaker in a neutron-rich system. Third, the isospinindependent part of the nuclear equation of state is attractive at low densities. Since this effect is proportional to the total surface area of the system, it increases rapidly with increasing thickness of the colliding nuclei [11]. For neutron-rich nuclei, the nucleon density distribution is more extended as shown in Fig. 1 and Fig. 2. Therefore, the isospin-independent attractive interaction is stronger in the neutron-rich system. Finally, the symmetry potential is generally repulsive. One expects a stronger effect of the symmetry potential in neutron-rich systems in which larger differences between neutron and proton densities exist. Although a more quantitative study on the relative importance of these mechanisms remains to be worked out, it is clear that the isospin-independent mean field plays a dominating role in causing the stronger negative deflection in neutron-rich systems. Moreover, the relative effects of these mechanisms depend strongly on the beam energy. As the beam energy increases the repulsive nucleon-nucleon collisions become dominant and effects of the neutron skin become less important. Also, the isospin dependence of the nucleon-nucleon cross sections becomes weaker at high energies [34]. It is therefore understandable that the isospin dependence of the collective flow disappears at high energies.

It is well known that the momentum-dependent interaction also affects significantly the 
transverse flow 18,26,44 46]. Most importantly, the momentum-dependent interaction gives more weight in terms of determining the collective flow to the mean field relative to the collision term. The observed stronger negative deflection in the neutron-rich system using the momentum-independent equation of state in Eq. 1 would therefore be further enhanced by the momentum-dependent interaction. Consequently, the balance energies in the two systems studied here would be even more separated, and this makes the isospin-dependence of the collective flow to be more easily observable. To quantitatively compare with forthcoming experimental data one thus needs to include carefully both the momentum- and isospin-dependence of the equation of state in transport models.

In summary, within the framework of an isospin-dependent BUU model using as inputs the neutron and proton density distributions calculated from the relativistic mean-field theory, we have demonstrated that there is a strong isospin dependence of the transverse collective flow. The reaction involving neutron-rich nuclei is found to have a significantly stronger attractive flow and consequently a higher balance energy compared to reaction systems having the same mass number but lower neutron/proton ratios. This isospin dependence is mostly easily observed in peripheral collisions at beam energies around and below the balance energy. Our study indicates that the isospin dependence of collective flow may provide a new approach to extract the isospin-dependent equation of state and to investigate properties of neutron-rich nuclei.

We would like to thank W. Bauer and G.D. Westfall for their suggestions and encouragement to carry out this study. We are also grateful to J.B. Natowitz, Gongou Xu, Zhongyu Ma and W. Mittig for helpful discussions. This work was supported in part by the NSF Grant No. PHY-9212209, PHY-9509266 and PHY-9457376, DOE Grant FG05-86ER40256 and the Robert A Welch Foundation under Grant A-1266. One of us (ZZR) was supported in part by grants from the Foundation of National Educational Commission of P.R. China and Ganil in France. One of us (SJY) also acknowledges the support from an NSF National Young Investigator Award. 


\section{REFERENCES}

[1] S. Das Gupta and G.D. Westfall, Physics Today, 46(5), 34 (1993).

[2] D. Krofcheck et al., Phys. Rev. Lett. 63, 2028 (1989).

[3] C.A. Ogilvie et al., Phys. Rev. C40, 2592; ibid, C42, R10 (1990);

Phys. Lett. B231, 35 (1989).

[4] J. Péter et al., Phys. Lett. B237, 187 (1990).

[5] J.P. Sullivan et al., Phys. Lett. B249, 8 (1990).

[6] J. Péter et al, Nucl. Phys. A519, 611 (1990).

[7] W.M. Zhang et al., Phys. Rev. C42, R491 (1990).

[8] D. Krofcheck et al., Phys. Rev. C43, 350 (1991); ibid 46, 1416 (1992).

[9] W.K. Wilson et al., Phys. Rev. C45, 768 (1992).

[10] G.D. Westfall et al, Phys. Rev. Lett. 71, 1986 (1993).

[11] D. Klakow, G. Welke and W. Bauer, Phys. Rev. C48, 1982 (1993).

[12] H. Stöcker and W. Greiner, Phys. Rep. 137, 277 (1986).

[13] G.F. Bertsch and S. Das Gupta, Phys. Rep. 160, 189 (1988).

[14] W. Bauer, C.K. Gelbke and S. Pratt, Ann. Rev. Nul. Part. Sci. 42, 77 (1992).

[15] P. Danielewicz and G. Odyniec, Phys. Lett. B157, 146 (1985).

[16] J. Molitoris and H. Stöcker, Phys. Lett. B162, 47 (1985).

[17] J. Molitoris, D. Hahn and H. Stöcker, Nucl. Phys. A447, 13c (1986).

[18] C. Gale, G.F. Bertsch and S. Das Gupta, Phys. Rev. C35, 1666 (1987).

[19] G.F. Bertsch, W.G. Lynch and M.B. Tsang, Phys. Lett. B189, 738 (1987). 
[20] M.B. Tsang, G.F. Bertsch, W.G. Lynch and M. Tohyama, Phys. Rev. C40, 1685 (1989).

[21] P. Danielewicz et al., Phys. Rev. C38, 120 (1989).

[22] V. de la Mota, F. Sebille, M. Farine, B. Remaud and P. Schuck, Phys. Rev. C46, 677 (1992).

[23] H.M. Xu, Phys. Rev. Lett. 67, 2769 (1992); Phys. Rev. C46, R392 (1992).

[24] Q. Pan and P. Danielewicz, Phys. Rev. Lett. 70, 2062 (1993).

[25] B.A. Li, Phys. Rev. C48, 2415 (1993).

[26] J. Zhang, S. Das Gupta and C. Gale, Phys. Rev. C50, 1617 (1994).

[27] S.J. Yennello et al., Phys. Lett. B321 (1994) 15.

[28] G.D. Westfall et al., NSCL/MSU proposal "Isospin dependence of transverse collective flow", 1995.

[29] K. Sumiyoshi, H. Suzuki and H. Toki, Report KEK-CP-031, RIKEN-AF-NP-95-59.

[30] C.J. Pethick and D.G. Ravenhall, in Neutron Stars: Theory and Observation, Eds. J. Ventura and D. Pines, NATO ASI Ser. C, Vol 344 (Kluwer, Dordrecht, 1991), P.3.

[31] G. Baym, in Neutron Stars: Theory and Observation, (Ref. [30]), p.21.

[32] B.A. Li and S.J. Yennello, Phys. Rev. C52, R1746 (1995).

[33] B.A. Li and J. Randrup, manuscript to be published.

[34] Total cross sections for reactions of high energy particles, A. Baldini, V. Flaminio, W.G. Moorhead and D.R.O. Morrison, (Springer-Verlag, Berlin), 1988.

[35] G.Q. Li and R. Machleidt, Phys. Rev. C49, 566 (1994).

[36] T. Alm, G. Röpke, W. Bauer, F. Daffin and M. Schmidt, Nucl. Phys. A587, 815 (1995).

[37] B.D. Serot and J.D. Walecka, Adv. Nucl. Phys. 16, 1 (1986). 
[38] M.M. Sharma, M.A. Nagarajan and P. Ring, Phys. Lett. B312, 377 (1993).

[39] M.M. Sharma, G.L. Lalazissis, W. Hillebrandt and P. Ring, Phys. Rev. Lett. 72, 1431 (1994).

[40] Zhongzhou Ren, W. Mittig, Baoqiu Chen and Zhongyu Ma, Phys. Rev. C52, R20 (1995).

[41] C.W. De Jager et al., Atomic Data and Nuclear Data Tables, 14, 479 (1974).

[42] L.G. Sobotka, Phys. Rev. C50, R1272 (1994).

[43] B. Jouault et al., Report Interne SUBATECH-95-11.

[44] C. Gale, G.M. Welke, M Prakash, S.J. Lee, and S. Das Gupta, Phys. Rev. C41, 1545 (1990).

[45] L.P. Csernai, G. Fai, C. Gale and E. Osnes, Phys. Rev. C 46, 736 (1992).

[46] V.K. Mishra, G. Fai, L.P. Csernai and E. Osnes, Phys. Rev. C47, 1519 (1993).

\section{FIGURE CAPTIONS}

Fig. 1 Proton (dot), neutron (dash) and matter (solid) density distributions in ${ }^{48} \mathrm{Ca}$ and ${ }^{58} \mathrm{Fe}$ calculated using the density-dependent relativistic mean-field theory.

Fig. 2 Same as Fig. 1 but for ${ }^{48} \mathrm{Cr}$ and ${ }^{58} \mathrm{Ni}$.

Fig. 3 Transverse momentum distributions in the reaction plane as a function of rapidity for reactions ${ }^{48} \mathrm{Ca}+{ }^{58} \mathrm{Fe}$ and ${ }^{48} \mathrm{Cr}+{ }^{58} \mathrm{Ni}$ at an impact parameter of $2 \mathrm{fm}$ and beam energies of 50,60 and $70 \mathrm{MeV} /$ nucleon.

Fig. 4 The flow parameter as a function of beam energy for reactions ${ }^{48} \mathrm{Ca}+{ }^{58} \mathrm{Fe}$ and ${ }^{48} \mathrm{Cr}+{ }^{58} \mathrm{Ni}$ at impact parameters of $2 \mathrm{fm}$ and $5 \mathrm{fm}$. The lines are the least-square fits to the calculations using linear functions. 
This figure "fig1-1.png" is available in "png" format from: http://arxiv.org/ps/nucl-th/9605015v1 
This figure "fig1-2.png" is available in "png" format from: http://arxiv.org/ps/nucl-th/9605015v1 
This figure "fig1-3.png" is available in "png" format from: http://arxiv.org/ps/nucl-th/9605015v1 
This figure "fig1-4.png" is available in "png" format from: http://arxiv.org/ps/nucl-th/9605015v1 